\title{
The Controlled Imitation Task: A new paradigm for studying self-other control
}

In the automatic imitation task (AIT) participants make a cued response during simultaneous exposure to a congruent or incongruent action made by another agent. Participants are slower to make the cued response in incongruent trials, which is thought to reflect conflict between the motor representation activated by the cue and the motor representation activated by the observed action. In incongruent trials, good performance requires the capacity to suppress the imitative action, in favor of producing the cued response. Here, we introduce a new experimental paradigm that complements the AIT, and is therefore a useful task for studying the control of self and other activated representations. In what we term the "Controlled Imitation Task (CIT)", participants are cued to make an action, but on 50\% of trials, within $100 \mathrm{~ms}$ of this cue, an on-screen hand makes a congruent or incongruent action. If the onscreen hand moves, the participant must suppress the cued response, and instead imitate the observed action as quickly and accurately as possible. In direct contrast to the AIT, the CIT requires suppression of a self-activated motor representation, and prioritization of an imitative response. In experiment 1, we report a robust pattern of interference effects in the CIT, such that participants are slower to make the imitative response in incongruent compared to congruent trials. In experiment 2, we replicate this effect while including a nonimitative spatial-cue control condition to show that the effect is particularly robust for imitative response tendencies per se. Owing to the essentially opposite control requirements of the CIT versus the AIT (i.e., suppression of self-activated motor representations instead of suppression of other-activated motor representations), we propose that this new task is a potentially informative complementary paradigm to the AIT that can be used in studies of selfother control processes. 
4 Social Brain, Body \& Action Lab, Centre for Cognitive Neuroscience \& Department of

5 Psychology, Wilfrid Laurier University, Waterloo, Ontario, Canada

6 Corresponding author: Sukhvinder Obhi (sobhi@wlu.ca)

\section{$7 \quad$ Abstract}


8 In the automatic imitation task (AIT) participants make a cued response during simultaneous

9 exposure to a congruent or incongruent action made by another agent. Participants are slower to

10 make the cued response in incongruent trials, which is thought to reflect conflict between the

11 motor representation activated by the cue and the motor representation activated by the observed

12 action. In incongruent trials, good performance requires the capacity to suppress the imitative

13 action, in favor of producing the cued response. Here, we introduce a new experimental paradigm

14 that complements the AIT, and is therefore a useful task for studying the control of self and other

15 activated representations. In what we term the "Controlled Imitation Task (CIT)", participants are

16 cued to make an action, but on $50 \%$ of trials, within $100 \mathrm{~ms}$ of this cue, an on-screen hand makes

17 a congruent or incongruent action. If the onscreen hand moves, the participant must suppress the

18 cued response, and instead imitate the observed action as quickly and accurately as possible. In

19 direct contrast to the AIT, the CIT requires suppression of a self-activated motor representation,

20 and prioritization of an imitative response. In experiment 1 , we report a robust pattern of

21 interference effects in the CIT, such that participants are slower to make the imitative response in

22 incongruent compared to congruent trials. In experiment 2, we replicate this effect while

23 including a non-imitative spatial-cue control condition to show that the effect is particularly

24 robust for imitative response tendencies per se. Owing to the essentially opposite control

25 requirements of the CIT versus the AIT (i.e., suppression of self-activated motor representations

26 instead of suppression of other-activated motor representations), we propose that this new task is

27 a potentially informative complementary paradigm to the AIT that can be used in studies of self-

28 other control processes.

29 Keywords: Imitation, Automatic Imitation, Controlled Imitation, Mirror System, Motor

30 Resonance, Automatic Imitation Task, Self-related processing, Other-related processing, Self-

31 other control 
$\underline{\text { Introduction }}$

33 In a popular version of the automatic imitation task (AIT), participants respond to a

34 symbolic cue (usually a number) that instructs them to lift either their index finger or their middle

35 finger. The symbolic cue is overlaid on a video showing another individual lifting their index or

36 middle finger. When the cue and the video are incongruent, there is a reaction time (RT) cost to

37 produce the cued movement compared to when the cue and the video are congruent. This cost,

38 termed "interference" is thought to be due to automatic activation of motor representations

39 matching the observed action. Numerous control studies and conditions have been employed to

40 demonstrate that the task really does seem to isolate automatic imitative tendencies and not

41 simply spatial compatibility or other confounding processes (Brass, Bekkering, Wohlschläger, \&

42 Prinz, 2000; see Heyes, 2011 for a review), though the extent to which they are etiologically

43 similar is a matter of open debate (Bertenthal \& Scheutz, 2013; Cooper, Catmur, \& Heyes, 2012).

44 In essence, the AIT assesses the degree to which an observer's motor system 'resonates'

45 with an observed action of another individual. Successful task performance in the incongruent

46 condition specifically involves suppressing the automatic tendency to imitate another individual,

47 in favor of making the cued response (Heyes, 2011; Santiesteban, White, et al., 2012). The

48 difference between incongruent and congruent trial RT's is robust even when participants expect

49 the observed action to contradict their response, and thus the paradigm can be considered within

50 the set of cognitive tasks that are generally accepted as reflecting automatic processes, such as the

51 Stroop task (Hogeveen \& Obhi, 2013; Stroop, 1935; Tzelgov, Henik, \& Berger, 1992). Notably

52 however, the neurocognitive bases for automatic imitation has been dissociated from Stroop

53 interference, as neuroimaging and lesion data have shown that the two tasks are associated with

54 activity in largely different brain regions (Brass, Derrfuss, \& von Cramon, 2005; Brass, Zysset, \&

55 von Cramon, 2001; Spengler, von Cramon, \& Brass, 2010). 
At the neural level, the automatic tendency to imitate the actions of another agent is

57 thought to be due to mirror activity which induces motor resonance - the specific activation of motor representations that would be active if the observer themselves performed the action they are watching (Hogeveen \& Obhi, 2011). This idea is bolstered by evidence from transcranial magnetic stimulation (TMS) studies which have consistently found an increase in motor cortical excitability when a participant watches an action, that is specific to the motor representation that would be involved in the performance of the action (e.g. Fadiga, Fogassi, Pavesi, \& Rizzolatti, 1995).

Over recent years the AIT, in one form or another, has been heavily employed by researchers interested in social information processing in normal participants and in participants with a range of disorders, but most notably autism spectrum disorders (ASD)(Cook \& Bird, 2012; Hogeveen \& Obhi, 2011; Spengler, Bird, \& Brass, 2010; Wang, Ramsey, \& Hamilton, 2011). A strong suggestion has been made that a key process involved in the AIT is the process of controlling self-related and other-related representations (Brass, Ruby, \& Spengler, 2009; Obhi, Hogeveen, Giacomin, \& Jordan, in press; Santiesteban, White, et al., 2012; Spengler, Bird, et al., 2010). Indeed, in a recent study, Santiesteban and colleagues (2012) asked a group of participants to perform the opposite movement from the model onscreen for an extensive training period

73 (counter-imitation training). The next day, they found a downstream improvement in both i) their

74 performance on incongruent trials of the automatic imitation task, and ii) their ability to take

75 someone else's visual perspective. In the perspective taking task participants were required to move objects to locations within a bookshelf as requested by another person on the other side of

77 the bookshelf, counter-imitation training was found to improve participants' ability to suppress their own perspective and take the perspective of the other. On the one hand, reduced interference in the AIT corresponds to suppressing influence from another agent in favor of prioritizing a more task relevant cued response. On the other hand, taking the perspective of another person involves 
81 suppressing the self-related representation, in favor of the other perspective. In this light, both

82 tasks can be said to require control of self-related and other-related representations.

The AIT, then, is an example of a task requiring self-other control processes and in

84 particular, the control requirement in the critical incongruent trials is to suppress the automatic

85 imitative response, and enforce, or prioritize the cued response. In the present study, we introduce a complementary paradigm for the study of self-other control which has essentially the opposite control requirements to the AIT. We term the paradigm the "controlled imitation task" (CIT) so as to contrast it explicitly with the "automatic imitation task". In the CIT, as in the AIT, participants are exposed to a picture of a neutral hand upon which a symbolic cue in the form of a numeral is superimposed. Also akin to the AIT, the participant must lift their index finger in response to the number 1 and must lift their middle finger in response to the number 2. Critically, however, both the temporal evolution of a given trial, and the task instructions in the CIT clearly dissociate it

93 from the AIT. With respect to trial timing, whereas the AIT cue and movement appear

94 simultaneously, in the CIT the cue is presented before the onset of any observed movement. Then,

95 within $100 \mathrm{~ms}$ of the cued response, the hand upon which the symbolic cue was presented makes

96 either a congruent or an incongruent action, such that half the time, participants see an index

97 finger lift when they are preparing an index finger lift (congruent trial), or they see a middle

98 finger lift when they are preparing an index finger lift (incongruent trial), and vice versa for cued

99 middle finger lifts. With respect to task instructions, whereas in the AIT participants are asked to

100 ignore the observed movement and enforce the cued response, in the CIT if the onscreen finger

101 moves participants are instructed to respond immediately with an identical finger movement. In

102 essence, on incongruent trials of the CIT participants must suppress their own cued action

103 preparation, in favor of producing the imitative response - which constitutes the opposite control

104 requirement compared to the standard AIT. Crucially, these congruent and incongruent trials only

105 occur on half of the total trials in a block, and in the other half of trials, the neutral hand on the 
106 107 participants expect to respond to the cue, rather than simply waiting for an imperative movement 108 to occur.

109

110

screen does not move and participants simply execute the cued response. Thus, across the block,

Using this paradigm, we predicted that the RT to make the controlled imitative response would be greater in incongruent trials compared to congruent trials. However, in contrast to the RT cost in the AIT, which is driven by the need to suppress imitation, the RT cost in the CIT would reflect the time required to suppress the cued response, in favor of producing an imitative response. If the congruence effects we predict are found, we suggest that this task could be a useful paradigm for the study of self-other control processes. In particular, the use of the CIT in conjunction with the AIT, in experiments where self-other control is manipulated in the context of imitation (Santiesteban, Banissy, Catmur, \& Bird, 2012; Santiesteban, White, et al., 2012), would allow researchers to be confident that what they are manipulated is indeed control of co-activated self- and other-generated motor representations. Beyond this, we suggest that the CIT could be a useful paradigm for the more general study of processing relating to self and other.

\section{Experiment 1}

The aim of experiment 1 was to provide a proof of concept that an activated motor plan interferes with one's ability to imitate an incongruent observed action. To that end, participants planned to make simple index and middle finger lifts in response to the number 1 or 2 , respectively. During the response preparation period $(50,70$, or $90 \mathrm{~ms}$ after cue-onset), an onscreen hand was most likely to remain still (baseline condition), or might have depicted an index or middle finger lift. On one subset of trials, the depicted action was congruent with the symbolic cue, and was therefore congruent with the participants' motor plan. However, on another set of trials, the depicted action was incongruent with the symbolic cue, and participants had to modify their plan to prioritize execution of an imitative response. The magnitude of the 
performance difference between the congruent and incongruent conditions could be informative

131 about the degree to which a symbolically cued motor plan can interfere with the execution of an

132 imitative action.

133 Methods

134 Participants

135 Twelve participants ( 9 female) between the ages of 19 and $33(M=23.33, S D=3.55)$

136 completed the study. Three of the 12 participants were left-handed, and all had normal or

137 corrected-to-normal vision. The study conformed to local ethical guidelines and informed consent

138 was obtained from all participants.

\section{Stimuli \& Apparatus}

140 The experiment was programmed using Superlab v.4.5 (Cedrus Corporation) and run on a

141 Lenovo desktop computer (Lenovo Group Limited). Experimental stimuli were adapted from

142 previous automatic imitation studies (Brass et al., 2000; Cook \& Bird, 2012; Hogeveen \& Obhi,

143 2013). The stimuli contained a number cue, followed by an image series depicting either an index

144 or middle finger movement (congruent and incongruent conditions), or a still image of a hand

145 (baseline; Figure 1). Baseline trials made up 50\% of the total trials, to ensure that participants

146 were expecting to make the cued response and not simply waiting for the movement of the

147 onscreen hand. The images were rotated orthogonally to the participants' hand to mitigate the

148 influence of spatial compatibility between the observed action and the appropriate response. On

149 baseline trials, the hand stayed in the same position for the duration of the trial (568ms). On

150 congruent and incongruent trials, the still hand was replaced by a number cue, which was then

151 replaced by the first stage $(34 \mathrm{~ms})$, second stage $(34 \mathrm{~ms})$, and final stage $(500 \mathrm{~ms})$ of an index or

152 middle finger lift. The final event on each trial was a blue screen, which was presented for

$1533000 \mathrm{~ms}$ to allow for delayed responses and provide participants with enough time to get situated

154 for the next trial. 


\section{Procedure}

156 The experiment took place in a dimly lit cubicle. Participants were seated at the computer,

157 told they would be holding down the $v$ and $b$ keys of the keyboard with their right index and

158 middle fingers throughout the experiment, and would need to execute an index or middle finger

159 lift as fast and accurately as possible when the number 1 or 2 was presented onscreen,

160 respectively. Responses were made by releasing the $v$ or $b$ keys on a standard Lenovo keyboard.

161 Further, participants were told: "if the hand onscreen moves, cancel your response and imitate

162 what you see regardless of the movement you were planning to make."

163 The study began with a practice session containing 24 trials, which observed by the experimenter

164 to ensure that participants understood the task. If the participant made more than 5 mistakes, the

165 practice section was re-run, which only occurred for one participant in the sample. The

166 experiment proper was divided into six blocks of 32 trials, each containing four congruent index

167 finger trials, four congruent middle finger trials, four incongruent index finger trials, four

168 incongruent middle finger trials, eight baseline index finger trials, and eight baseline middle

169 finger trials. Each trial began with a still image of the hand on screen $(300,400,500,600,700$, or

$170800 \mathrm{~ms})$, then the response cue was presented, and after a delay period $(50,70$, or $90 \mathrm{~ms})$ the hand

171 either moved or stayed still (see Stimuli \& Apparatus for a description of the hand movement).

$172 \underline{\text { Results }}$

173 Data Exclusion

174 As described in the Methods section, the baseline condition was in place purely to ensure

175 that participants actually prepared the cued response on each trial, and was excluded from further

176 analysis. Reaction time data for index and middle finger actions were collapsed and responses

177 that were 3 SD's above or below the mean within each experimental condition (i.e. congruent, or 
178 incongruent) were excluded from statistical analysis, resulting in the removal of $0.39 \%$ of the

179 data. The remaining congruent and incongruent trial data was then subjected to inferential

180 statistical analysis.

181 Reaction Time (RT)

182 Each participant's average RT for correct responses was entered into a repeated-measures

183 ANOVA with one factor (congruence: congruent, or incongruent). The effect of congruence was

184 highly significant $\left(F_{1,11}=103.06, p<.001, \eta^{2}=.90\right)$. Participants were faster on congruent trials

$185(M=434, S E=9)$ than they were on incongruent trials $\left(M=553, S E=14 ; t_{11}=-10.15, p<.001, \eta^{2}=.68\right.$;

186 Figure 2A).

187 Proportion of Errors (PE)

188 Errors and missed responses (3.95\% of total data) were summed and divided by the

189 number of trials, yielding the proportion of errors (PE) for each participant for each condition.

190 This data was entered into a repeated-measures ANOVA akin to the RT data, and the main effect

191 of congruence was highly significant $\left(F_{1,11}=38.11, p<.001, \eta^{2}=.78\right)$. Participants made a

192 substantially smaller number of errors on congruent $(M=0.01, S E=0.01)$ trials compared to

193 incongruent trials $\left(M=0.14, S E=0.02 ; t_{11}=-6.17, p<.001, \eta^{2}=.46\right.$; Figure 2B).

194 Discussion

195 The RT interference effect in the well-studied AIT indexes the cost associated with

196 inhibiting other-related processing in favor of executing a self-related task. In study 1, we

197 establish the CIT as a potential method for measuring the cost of self-related motor preparation to

198 the execution of a conflicting imitative response - i.e. the cost of inhibiting self-related 
processing to prioritize processing of the other. Specifically, we found a robust effect in the CIT task: participants were $119 \mathrm{~ms}$ slower on incongruent relative to congruent trials. While this result

201 provides reason to believe that the CIT might be a useful addition to the study of self-other

202 related processing, it is possible that the results simply represent the cost of action modification

203 generally, rather the cost associated with conflicting self-other representations per se. It is

204 worthwhile pointing out that the size of the CIT effect $(119 \mathrm{~ms})$ is much greater than we would be 205 anticipate in response to a socially innocuous action modification cue (e.g. 40ms for an auditory 206 tone; Obhi, Matkovich, \& Gilbert, 2008), suggesting that the robust effects reported in

207 experiment 1 were at least partially driven by the 'specialness' of the observed action. Regardless,

208 we conducted a second experiment to assess the extent to which the CIT reflects response

209 modification generally, or is specific to controlling co-activated self-other motor representations.

210 To shed more light on how the CIT and AIT might be useful complementary paradigms, we also

211 added a set of AIT blocks to experiment 2.

\section{Experiment 2}

In experiment 2, we sought to determine whether the CIT indexes self-other control in an

214 imitation context, or simply quantifies the cost associated with modifying a planned response. To

215 this end, participants performed the same task as described in experiment 1, but with the addition

216 of a moving dot condition (spatial control; Cross, Torrisi, Losin, \& Iacoboni, 2013; Figure 3). The

217 dot cues were superimposed over the hand stimuli from experiment 1 , and on CIT blocks

218 participants were instructed to match what they saw onscreen whenever one of the dots, or one of

219 the fingers, moved during their response preparation period. The difference between 'finger

220 incongruent-congruent' interference and 'dot incongruent-congruent' interference provides an

221 approximation of the cost associated with cancelling a self-related motor plan in favor of an 
222

\section{3}

224

225

226

227

228

229

230

231

232

233

234

235

236

237

238

imitative response, although as with all subtractions, this method may not perfectly isolate imitative processing. Experiment 2 also included a set of AIT blocks.

$\underline{\text { Methods }}$

\section{Participants}

Sixteen participants ( 9 female) between the ages of 17 and $40(M=22.44, S D=5.18)$

completed the study. All of the participants were right-handed, and had normal or corrected-tonormal vision. The study conformed to local ethical guidelines and informed consent was obtained from all participants.

\section{Stimuli \& Apparatus}

Experimental stimuli \& apparatus were similar to experiment 1, with two exceptions: i) black dots were superimposed over the finger-nails of each finger, and ii) on trials where the finger or dot moved, the movement occurred at once, rather than containing intermediate movement stages (cf. Catmur \& Heyes, 2011; Press, Bird, Flach, \& Heyes, 2005)(Figure 3). On trials where the fingers moved, the dots remained in position; whereas on trials where the dots moved the fingers remained in position. The final locations of the dots in the spatial control conditions were positioned at the final locations of the finger movements in the imitative conditions.

Procedure

The CIT and AIT each contained five experimental conditions randomized within blocks: congruent dot, incongruent dot, congruent finger, incongruent finger, and baseline (Figure 3). The

242 trial events in the CIT were nearly identical to experiment 1, except experiment 2 contained a

243 longer neutral stimulus and a shorter blue screen. Participants completed a practice CIT block (32

244 trials: 4 congruent dot, 4 incongruent dot, 4 congruent finger, 4 incongruent finger, 16 baseline) 
245 followed by experimental CIT blocks ( 4 blocks x 48 trials) that contained a total of 24 trials per

246 operant (i.e. congruent, incongruent) condition. Akin to experiment 1, on the CIT blocks

247 participants were instructed: "if the hand or the dot onscreen moves, cancel your response and

248 match what you see regardless of the movement you were planning to make." Participants who

249 made $>5$ errors during the practice block in the CIT $(n=4)$ performed the practice twice.

In experiment 2, participants completed a practice AIT block and four experimental AIT

blocks containing the same randomized trial type distributions as the CIT. The order of

252

presentation for the two tasks was counterbalanced across the sample. Prior to the AIT,

participants were instructed to perform index and middle finger lifts in response to the numbers 1

or 2, respectively, and to execute that plan "no matter what the hand or dot onscreen does." Thus,

overall the stimuli and task demands required for the AIT and CIT were identical, except in the

former enforcing self was required to inhibit other, whereas in the latter enforcing other was

required to imitate and cancel a planned response.

258 Results

\section{Data Exclusion}

260 As in experiment 1, the baseline condition was in place to ensure that participants

264 from the mean was violated by one participant's mean RT in the congruent finger condition, and

265 this participant was removed from the dataset (leaving $n=15$ ). It is worthwhile to note that the

266 subsequently reported inferential results were identical with or without the outlying participant. 
269 finger) x 2 (congruence: dot, finger) repeated measures ANOVA (rmANOVA). There was no

270 effect of stimulus type ( $p=.14)$, but the effect of congruence was highly significant $\left(F_{1,14}=80.95\right.$,

$\left.271 p<.001, \eta^{2}=.85\right)$. Most importantly, the CIT RT analysis yielded a significant interaction between

272 stimulus type and congruence $\left(F_{1,14}=6.00, p<.05, \eta^{2}=.30\right)$. To elucidate the interaction,

273 interference effects were calculated for the dot and finger conditions, which were then entered

274 into a rmANOVA with one factor (stimulus type: dot, finger). The mean interference effect for the

275 spatially-matched dot control $(M=74, S D=34)$ was significantly smaller than the interference

276 effect for the imitation condition $\left(M=98, S D=48 ; F_{1,14}=5.93, p<.05, \eta^{2}=.30\right)$.

277 Next, mean correct RT's on the AIT blocks were entered into a 2 (stimulus type: dot, finger) x 2

278 (congruence: dot, finger) rmANOVA. Neither of the main effects were significant ( $p \mathrm{~s}>.1)$, but

279 importantly the interaction between stimulus type and congruence was significant on the AIT

$280\left(F_{1,14}=8.21, p<.05, \eta^{2}=.37\right)$. Akin to the CIT analysis, the interference effects on the AIT were

281 computed and entered into a rmANOVA with one factor (stimulus type: dot, finger). Again, akin

282 to the CIT, the dot control $(M=7, S D=38)$ had a much smaller effect than the imitative condition

$283\left(M=30, S D=49 ; F_{1,14}=8.23, p<.05, \eta^{2}=.37\right)$ in the AIT context.

285 previous AIT studies have found a main effect of congruence even when a similar spatially-

286 matched control condition was included (Cook \& Bird, 2011, 2012). One possible reason for this

287 discrepancy could be that the order of the two tasks (i.e. CIT then AIT vs. AIT then CIT)

288 confounded congruence in the AIT. To isolate any order effects, the $2 \times 2$ rmANOVA's described

289 for the CIT and AIT were re-run as analyses of covariance (rmANCOVA), regressing out the task 
order term. Interestingly, the rmANCOVA's for the CIT ( $p=.19)$, and the AIT ( $p=.56)$, no longer

291 contained significant interaction effects. Participants who performed the AIT before the CIT

292 showed a significant interaction on the CIT, with dots $(M=78, S D=28)$ having a smaller

293 interference effect than fingers $\left(M=110, S D=54 ; F_{1,6}=6.06, p<.05, \eta^{2}=.50\right)$, whereas participants

294 who performed the CIT immediately did not show a stimulus type preference ( $p=.29)$.

295 Conversely, participants who performed the CIT first showed a significant stimulus type

296 preference on the AIT, with dots $(M=17, S D=46)$ having a smaller effect than fingers $(M=42$,

$297 S D=53 ; F_{1,7}=6.39, p<.05, \eta^{2}=.48$ ), and participants who performed the AIT immediately showing

298 no difference as a function of stimulus type $(p=.18)$.

299 Discussion

300 After documenting an large CIT interference effect in experiment 1, we conducted

301 experiment 2 in order to replicate the CIT, and to differentiate controlled imitation from response

302 modification by including a non-social cue condition. The results from experiment 2 demonstrate

303 again that the size of the RT cost associated with cancelling a self-related movement in favor of

304 imitation is quite large (98ms), and that it is greater than the cost in a well-matched spatial-cue

305 condition (dots: $74 \mathrm{~ms}$ ). The finding of a greater interference effect to the finger stimulus in both

306 the CIT and the AIT is likely due to the greater degree of overlap between the conflicting

307 representations relative to the moving dot conditions. Whereas the spatial location and movement

308 affordances are matched between the finger and dot stimuli, the former contains a

309 topographically congruent or incongruent movement by a model that is not present in the latter.

310 Without saying anything about their ontogeny (Cooper et al., 2012), the present finding is

311 consistent with suggestions that, once developed, imitative compatibility reflects a form of

312 stimulus-response compatibility that is unique, or at least more pronounced, than non-imitative 
313 spatial-cue-driven effects (Catmur \& Heyes, 2011). Further, we suggest that experiment 2 utilizes

314 a useful control condition for the CIT, which future studies attempting to manipulate imitative

315 self-other control should include to differentiate controlled imitation from general response

316 modification processes.

\section{General Discussion}

318 In two experiments, we report a novel task requiring the online control of competing self-

319 and other-generated motor representations. We have called this task the controlled imitation task

320 (CIT), and suggest that it is a useful complementary task to the well-studied AIT. On each

321 experimental trial, participants had to prepare a cued response, but on $50 \%$ of trials they were

322 required to suppress this response and instead prioritize the production of an imitative response

323 that was either congruent or incongruent with the cue. The robust result is that participants were

324 significantly better at imitating an observed movement when it matched the response cue, relative

325 to when it did not match the cue (experiment 1), and that this effect was reliable and of a much

326 larger magnitude than the traditional AIT (experiment 2).

327 We suggest the CIT provides a valuable method for examining the influence of the self

328 with respect to self-other control in the imitative context. To the extent that the production of a

329 symbolically-cued response reflects a task specific self-activated process, the incongruent

330 condition in the CIT can be thought of as requiring suppression of this process in favor of

331 producing an imitative response. Thus, the present nicely complement typical AIT data: in the

332 AIT interference effects reflect the cost of inhibiting an other-activated motor representation in 
333 favor of a cued response, whereas in the CIT interference effects reflect the cost of inhibiting a

334 self-activated motor representation in favor of imitation.

336 more pronounced than general response modification and inhibition, respectively, but also that

337 this biological preference is driven by recent experience. Typical demonstrations of biological-

338 specificity assume they are present a priori (Kanwisher, McDermott, \& Chun, 1997; Kilner,

339 Paulignan, \& Blakemore, 2003), but biological-specificity in the present experiment varied as a

340 function of task order. Participants who engaged in the CIT initially, showed a finger-preference

341 on the subsequent AIT, and participants who did the AIT first showed this preference on the

342 subsequent CIT, but neither group showed a stimulus preference during the initial task. This

343 suggests that inhibiting imitation at time 1 (AIT) makes it more difficult to perform imitation at

344 time 2 (CIT), which was not true for the dot control condition. Conversely, performing imitation

345 at time 1 (CIT) makes it harder to inhibit imitation at time 2 (AIT), which again was not the case

346 for the dot control condition. Thus, it appears the shared self-other representational system that is

347 invoked to explain the traditional AIT is also engaged by the CIT. Furthermore, this system

348 seems to be strongly affected by recent experience (Catmur, Walsh, \& Heyes, 2007; Heyes, Bird,

349 Johnson, \& Haggard, 2005; Hogeveen \& Obhi, 2012).

351 possibilities for future work that could be studied with the CIT. Specifically, in light of a recent

352 training study designed to improve self-other control (Santiesteban, White, et al., 2012), the CIT

353 provides an excellent and quite well matched experimental paradigm with which to contrast AIT,

354 and investigate task-appropriate shifts in processing towards self or other in the motor domain.

355 Their data demonstrate that imitation-inhibition training on day 1 suppresses the automatic 
tendency to imitate on day 2 , in order to facilitate task performance which necessitates production

357 of the cued response. Whereas the size of the AIT interference effect was reduced after training,

358 accuracy on a visual perspective-taking task was improved, suggesting that processing of the

359 other person was enhanced or inhibited in a task-appropriate manner (Santiesteban, White, et al.,

360 2012). Despite the impressive nature of this finding, the CIT provides a closer other-centric

361 homologue for AIT than visual perspective taking. Thus, if counter-imitation training has a

362 functional impact on task dependent control of self- and other-generated motor representations,

363 we would expect to observe an increased ability to suppress the self-activated motor

364 representation in the CIT. Thus, the control process in the CIT, at least in the incongruent trials,

365 requires the suppression of a self-related representation whereas the control process in the

366 standard AIT requires the suppression of an other-related representation. Seeing a pattern of

367 results that is consistent with suppression of self-related activity in the CIT combined with

368 suppression of other-related activity in the AIT would bolster the notion that the counter-imitation

369 training improves task specific control of self-other processing.

In addition to comparing the AIT and CIT directly after a self-other control manipulation,

the extant difference between the two tasks could be used to index individual differences in self-

related bias. On their own, the size of the CIT or AIT interference effects may reflect self-other

distinction, control, or both. However, by subtracting the AIT from the CIT, experimenters could quantify the extent to which self-other control is biased in favor of the self, which could then be related to personality variables hypothesized to relate to self-other control. It remains for future work to investigate this suggestion further. 
379 against external cueing of action in non-imitative contexts (Obhi \& Haggard, 2004; Obhi,

380 Matkovich, \& Chen, 2009; Obhi et al., 2008). In particular, in the truncation paradigm

381 participants begin the trial preparing to make an index finger movement at a time of their own

382 choosing, but are interrupted on a portion of trials by a tone prompting production of the same

383 movement. That, is, in the truncation paradigm, participants prepare to make a self-paced action

384 and are interrupted with a non-social external cue to produce the same action or a different action,

385 whereas in the current study the external cue to action is a movement by another person. Given

386 the dissociation between the ability to inhibit imitation in the AIT and the inhibition of other

387 overlearned responses (Brass et al., 2005; Spengler, von Cramon, et al., 2010), it would be

388 interesting to determine whether the CIT and action modification in the truncation paradigm

389 depend on similar or specialized neural substrates. Implementation of this line of enquiry would

390 necessitate modification to the CIT to include a self-paced action, as opposed to a cued action.

391 Such experiments could be useful for understanding the domain-generality or domain-specificity

392 of the functional mechanisms underlying self-other control.

393 In summary, in two experiments we introduce a new paradigm that we have termed the

394 "controlled imitation task". Using an identical stimulus set to the established AIT, the CIT yields

395 robust interference and facilitation effects that appear to shed light on self-other control

396 processes, and that specifically tap into the capacity to suppress, or harness self-activated motor

397 representations to facilitate fast production of an other-activated response (cf. Brass et al., 2009).

398 We have outlined just two possible areas of inquiry where the CIT could a useful paradigm; self-

399 other control, and the more general exploration of internally versus externally triggered action

400 generation. It remains for future work to consider exactly how this paradigm can be employed to

401 further understanding in these areas of study. 
403

404

405

406

407

408

409

410

411

412

413

414

415

416

417

418

419

420

421

422

423

424

425

426

427

428

429

430

431

432

433

434

435

436

437

438

439

440

441

442

443

444 445

Bertenthal, B. I., \& Scheutz, M. (2013). In praise of a model but not its conclusions: commentary on Cooper, Catmur, and Heyes (2012). Cognitive Science, 37(4), 631-41; discussion 642-5. doi:10.1111/cogs.12039

Brass, M., Bekkering, H., Wohlschläger, A., \& Prinz, W. (2000). Compatibility between observed and executed finger movements: Comparing symbolic, spatial, and imitative cues. Brain and Cognition, 44(2), 124-143. doi:10.1006/brcg.2000.1225

Brass, M., Derrfuss, J., \& von Cramon, D. Y. (2005). The inhibition of imitative and overlearned responses: A functional double dissociation. Neuropsychologia, 43(1), 89-98. doi:10.1016/j.neuropsychologia.2004.06.018

Brass, M., Ruby, P., \& Spengler, S. (2009). Inhibition of imitative behaviour and social cognition. Philosophical Transactions of the Royal Society of London. Series B, Biological sciences, 364(1528), 2359-2367. doi:10.1098/rstb.2009.0066

Brass, M., Zysset, S., \& von Cramon, Y. (2001). The inhibition of imitative response tendencies. NeuroImage, 14, 1416-1423.

Catmur, C., \& Heyes, C. (2011). Time course analyses confirm independence of imitative and spatial compatibility. Journal of Experimental Psychology: Human Perception and Performance, 37(2), 409-421. doi:10.1037/a0019325

Catmur, Caroline, Walsh, V., \& Heyes, C. (2007). Sensorimotor learning configures the human mirror system. Current Biology, 17(17), 1527-31. doi:10.1016/j.cub.2007.08.006

Cavallo, A., Heyes, C., Becchio, C., Bird, G., \& Catmur, C. (2013). Timecourse of mirror and counter-mirror effects measured with transcranial magnetic stimulation. Social Cognitive and Affective Neuroscience, in press. doi:10.1093/scan/nst085

Cook, J. L., \& Bird, G. (2011). Social attitudes differentially modulate imitation in adolescents and adults. Experimental brain research. Experimentelle Hirnforschung. Expérimentation cérébrale, 211(3-4), 601-12. doi:10.1007/s00221-011-2584-4

Cook, J. L., \& Bird, G. (2012). Atypical social modulation of imitation in autism spectrum conditions. Journal of Autism and Developmental Disorders, 42(6), 1045-1051. doi:10.1007/s10803-011-1341-7

Cooper, R. P., Catmur, C., \& Heyes, C. (2012). Are automatic imitation and spatial compatibility mediated by different processes? Cognitive Science, 37(4), 605-630. doi:10.1111/j.15516709.2012.01252.x

Cross, K. A., Torrisi, S., Losin, E. A. R., \& Iacoboni, M. (2013). Controlling automatic imitative tendencies: Interactions between mirror neuron and cognitive control systems. NeuroImage, 83, 493-504. doi:10.1016/j.neuroimage.2013.06.060

Fadiga, L., Fogassi, L., Pavesi, G., \& Rizzolatti, R. (1995). Motor facilitation during action observation: A magnetic stimulation study. Journal of Neurophysiology, 73(6), 2608-2611.

Heyes, C. (2011). Automatic imitation. Psychological Bulletin, 137(3), 463-83. doi: $10.1037 / \mathrm{a} 0022288$

Heyes, C., Bird, G., Johnson, H., \& Haggard, P. (2005). Experience modulates automatic imitation. Brain Research, 22(2), 233-240. doi:10.1016/j.cogbrainres.2004.09.009

Hogeveen, J., \& Obhi, S. S. (2011). Altogether now: Activating interdependent self-construal induces hypermotor resonance. Cognitive Neuroscience, 2(2), 74-82.

doi:10.1080/17588928.2010.533164

PeerJ reviewing PDF | (v2013:07:696:1:0:NEW 23 Aug 2013) 
446

447

448

449

450

451

452

453

454

455

456

457

458

459

460

461

462

463

464

465

466

467

468

469

470

471

472

473

474

475

476

477

478

479

480

481

482

483

484

485

486

487

488

Hogeveen, J., \& Obhi, S. S. (2012). Social interaction enhances motor resonance for observed human actions. The Journal of Neuroscience, 32(17), 5984-5989. doi:10.1523/JNEUROSCI.5938-11.2012

Hogeveen, J., \& Obhi, S. S. (2013). Automatic imitation is automatic, but less so for narcissists. Experimental brain research. Experimentelle Hirnforschung. Expérimentation cérébrale, 224(4), 613-21. doi:10.1007/s00221-012-3339-6

Kanwisher, N., McDermott, J., \& Chun, M. M. (1997). The Fusiform Face Area : A Module in Human Extrastriate Cortex Specialized for Face Perception. The Journal of Neuroscience, 17(11), 4302-4311. Retrieved from http://www.ncbi.nlm.nih.gov/pubmed/9151747

Kilner, J., Paulignan, Y., \& Blakemore, S. (2003). An interference effect of observed biological movement on action. Current Biology, 13, 1274-1277. doi:10.1016/S

Obhi, S. S., \& Haggard, P. (2004). Internally generated and externally triggered actions are physically distinct and independently controlled. Experimental Brain Research., 156(4), 518-523. doi:10.1007/s00221-004-1911-4

Obhi, S. S., \& Hogeveen, J. (2010). Incidental action observation modulates muscle activity. Experimental Brain Research, 203(2), 427-435. doi:10.1007/s00221-010-2253-z

Obhi, S. S., Hogeveen, J., Giacomin, M., \& Jordan, C. H. (n.d.). Automatic imitation is reduced in narcissists. Journal of Experimental Psychology: Human Perception and Performance.

Obhi, S. S., Matkovich, S., \& Chen, R. (2009). Changing the "when" and "what" of intended actions. Journal of Neurophysiology, 102(5), 2755-2762. doi:10.1152/jn.00336.2009

Obhi, S. S., Matkovich, S., \& Gilbert, S. J. (2008). Modification of planned actions. Experimental Brain Research, 192(2), 299-299. doi:10.1007/s00221-008-1618-z

Press, C., Bird, G., Flach, R., \& Heyes, C. (2005). Robotic movement elicits automatic imitation. Cognitive Brain Research, 25(3), 632-640. doi:10.1016/j.cogbrainres.2005.08.020

Santiesteban, I., Banissy, M. J., Catmur, C., \& Bird, G. (2012). Enhancing social ability by stimulating right temporoparietal junction. Current Biology, 22(23), 2274-2277.

Santiesteban, I., White, S., Cook, J., Gilbert, S. J., Heyes, C., \& Bird, G. (2012). Training social cognition: from imitation to Theory of Mind. Cognition, 122(2), 228-35. doi:10.1016/j.cognition.2011.11.004

Spengler, S., Bird, G., \& Brass, M. (2010). Hyperimitation of actions is related to reduced understanding of others' minds in autism spectrum conditions. Biological Psychiatry, 68(12), 1148-1155. doi:10.1016/j.biopsych.2010.09.017

Spengler, S., von Cramon, D. Y., \& Brass, M. (2010). Resisting motor mimicry: Control of imitation involves processes central to social cognition in patients with frontal and temporoparietal lesions. Social Neuroscience, 5(4), 401-416. doi:10.1080/17470911003687905

Stroop, J. R. (1935). Studies of interference in serial verbal reactions. Journal of Experimental Psychology, 18, 643-662.

Tzelgov, J., Henik, A., \& Berger, J. (1992). Controlling Stroop effects by manipulating expectations for color words. Memory \& Cognition, 20(6), 727-735. Retrieved from http://www.ncbi.nlm.nih.gov/pubmed/1435275

Wang, Y., Ramsey, R., \& Hamilton, A. F. D. C. (2011). The control of mimicry by eye contact is mediated by medial prefrontal cortex. The Journal of Neuroscience, 31(33), 12001-12010. doi:10.1523/JNEUROSCI.0845-11.2011 
490 Figure 1. (A) Breakdown of the events in a trial of Experiment 1, displaying an incongruent

491 index finger movement trial as an example. (B) Breakdown of the different types of trials in

492 Experiment 1, that were randomized within blocks. Note: the number of baseline trials was equal

493 to the sum total of congruent and incongruent trials 
494 Figure 2. Performance data for Experiment 1, with (A) reaction time (RT), and (B) proportion of 495 errors (PE) plotted by experimental condition. Data labels represent the mean (and standard error 496 of the mean) for each bar. The congruence effect was highly significant for both RT and PE (all $497 p \mathrm{~s}<.001)$. 
498 Figure 3. Breakdown of the trial events and conditions from Experiment 2. (A) AIT displayed the

499 cue and finger or dot movement simultaneously, whereas (B) the CIT displays the cue, followed

500 by a finger or dot movement that appears during the response preparation period. (C) All of the

501 conditions that took place in the experiment, which were randomized within both the AIT and the

502 CIT blocks. Note: the cue numbers have been exaggerated for display purposes only. Also,

503 whereas Experiment 1 used picture sequences, Experiment 2 immediately moved to the final state

504 of the action, producing an apparent motion effect that has been shown to elicit robust effects in

505 past AIT studies (C. Catmur \& Heyes, 2011; Press et al., 2005). 
506 Figure 4. Size of the RT interference effect in experiment 2, with (A) the CIT and (B) the AIT

507 interference effects plotted by cue type. Data labels represent the mean (and standard error of the

508 mean) for each bar. The interference effect was significantly higher for the imitative cue in both

509 the CIT and the AIT tasks $(p s<.05)$. 


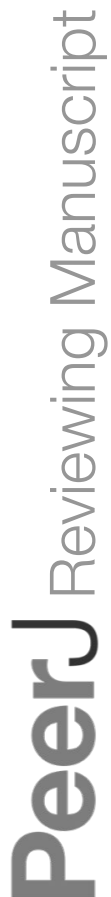

PeerJ reviewing PDF | (v2013:07:696:1:0:NEW 23 Aug 2013) 


\section{Figure 1}

\section{cit design}

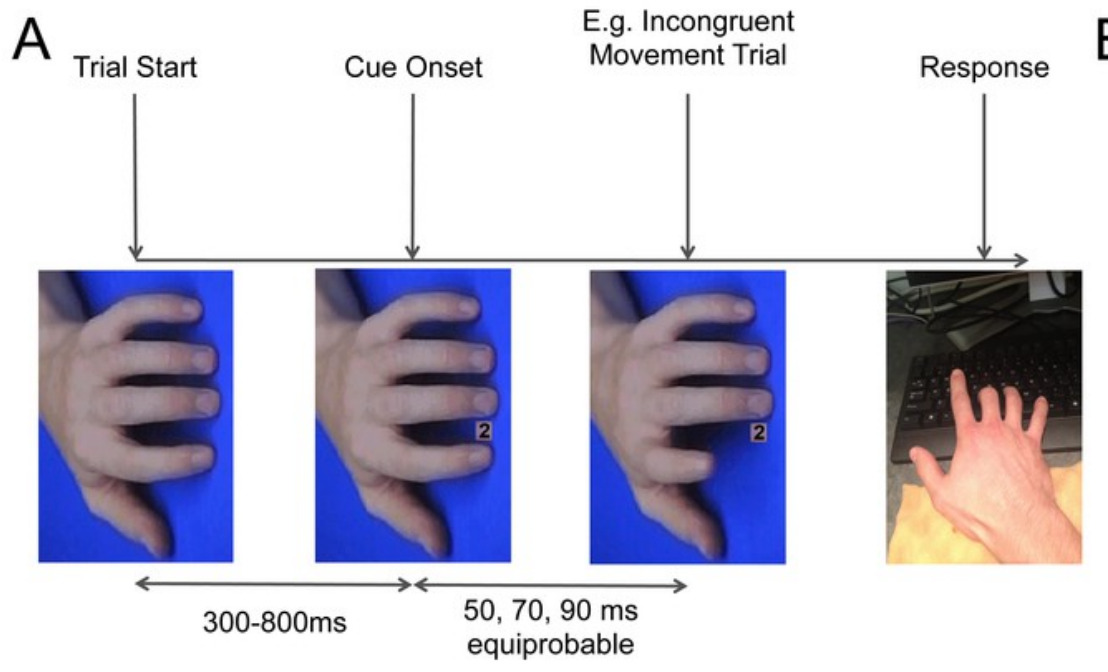

\section{B \\ Experimental Conditions}

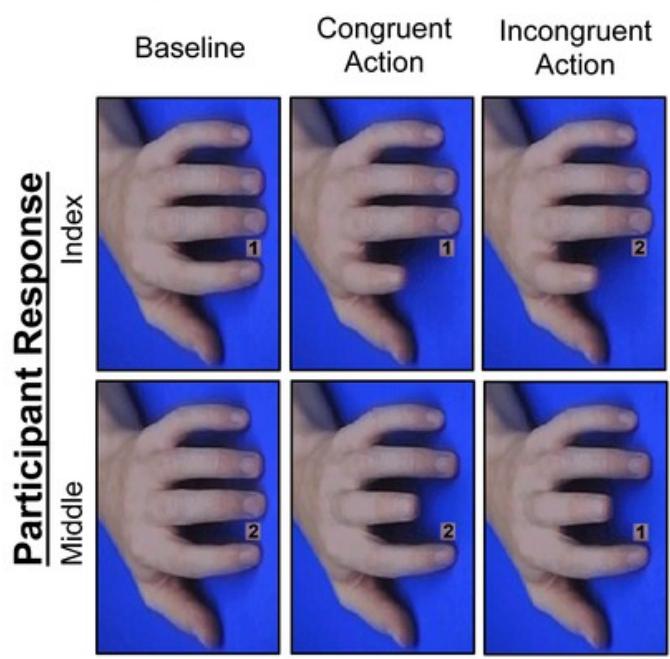




\section{Figure 2}

\section{cit results}

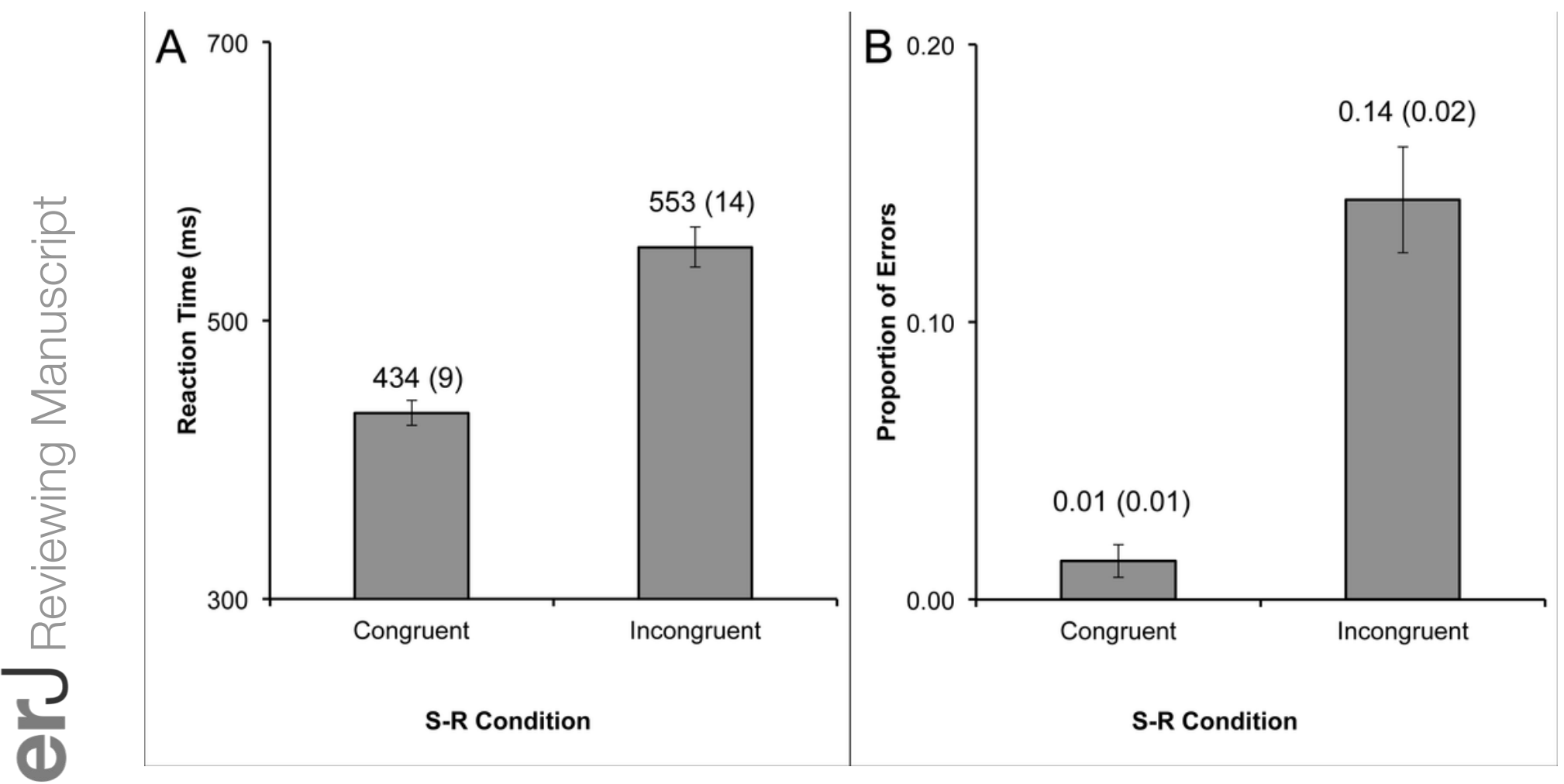




\section{Figure 3}

ait and cit design experiment 2

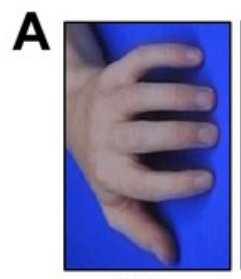

$800-$ $2400 \mathrm{~ms}$

B

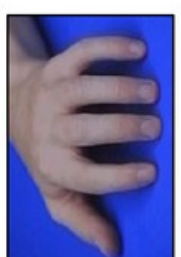

$800-$ $2400 \mathrm{~ms}$

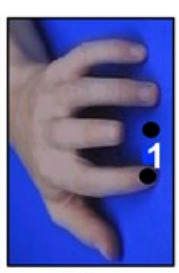

$568 \mathrm{~ms}$

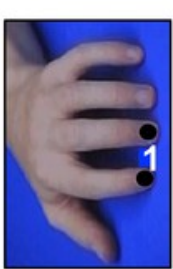

$50,70,90 \mathrm{~ms}$

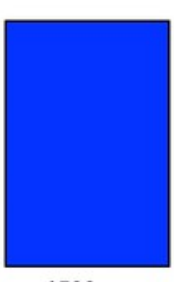

$1500 \mathrm{~ms}$

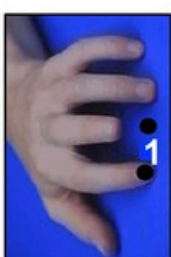

$568 \mathrm{~ms}$

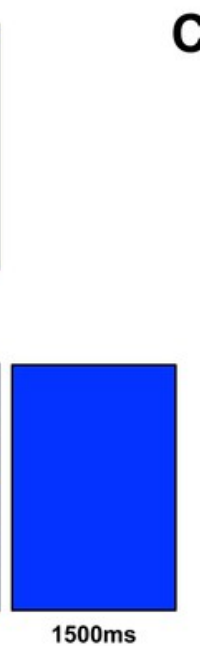

C

\section{Experimental Conditions}

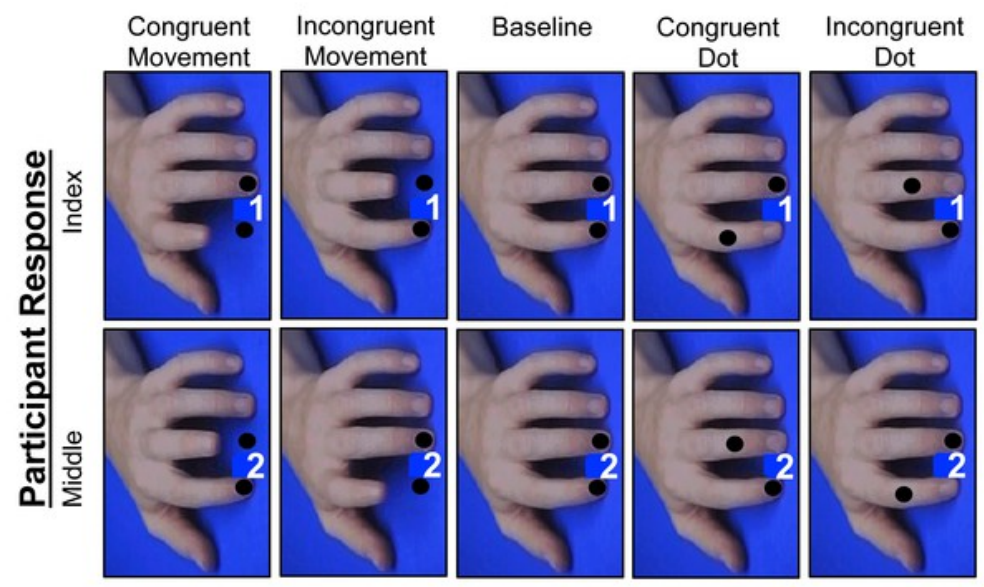




\section{Figure 4}

experiment 2 interference

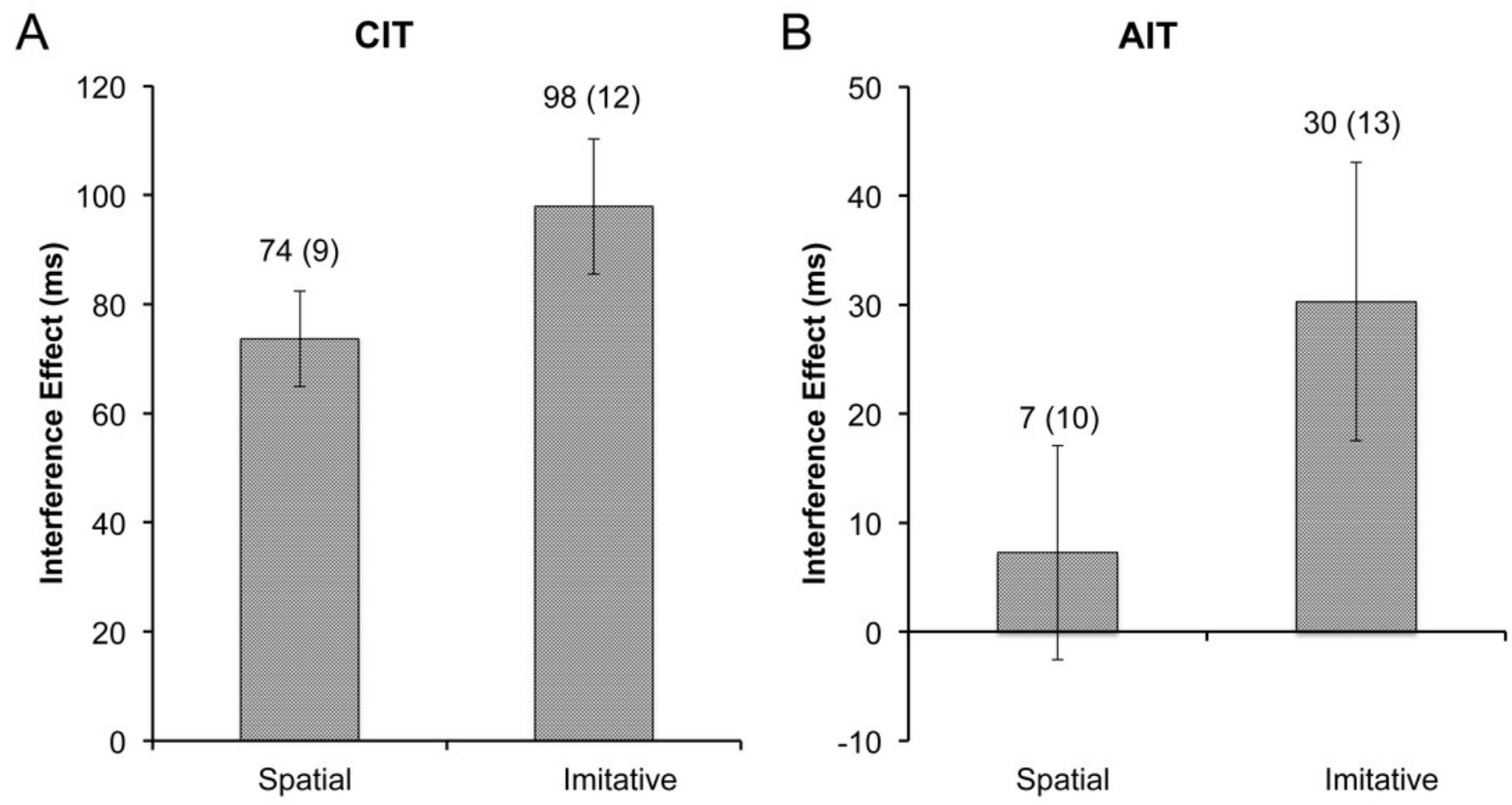

